\title{
What It's Cracked Up To Be
}

\author{
Researchers have designed a metamaterial that is nearly twice as resistant \\ to cracking in one direction versus the other-what they call a fracture \\ diode.
}

By Sophia Chen

A

Il known natural materials crack with equal likelihood in opposite directions, say from left-to-right and right-to-left. But artificial materials containing asymmetric microstructures can be designed to break more easily along a certain path. Now, Kaushik Bhattacharya of the California Institute of Technology and colleagues have designed and tested an artificial material with an asymmetric pattern that makes it nearly twice as tough in one direction than in the other [1].

The researchers' strategy consisted of sideways triangular voids with rounded corners arranged like a series of play button symbols across the material. The resulting metamaterial is tougher than the original material, but the increase in toughness is greater in one direction along the triangles than in the other. A crack entering a triangle at its point has difficulty penetrating further, as it needs to open a tear in the triangle's flat side. From the other direction, however, a crack can enter the triangle's flat side and relatively easily exit through its point and continue to the next triangle.

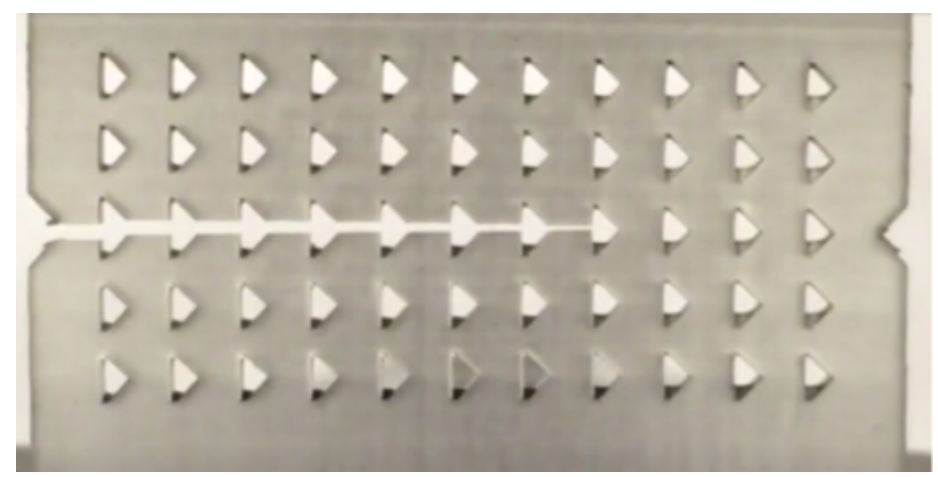

Credit: N. R. Brodnik et al. [1]
To determine the optimal shape and spacing of the triangles, the researchers used computational models that balanced two constraints: the triangles need to be close enough to guide a crack's path forward but far enough apart that a crack only spreads toward one triangle at a time. They tested these predictions in a 3D-printed piece of the plastic poly-methyl-methacrylate with the ideal hole pattern and found the material was twice as resistive to cracking in one direction than in the other. The researchers say that the insights from their metamaterial pave the way for new applications, in which unavoidable fracture can be guided through predesigned paths to minimize damage.

Sophia Chen is a freelance science writer based in Columbus, Ohio.

\section{REFERENCES}

1. N. Brodnik et al., "Fracture diodes: Directional asymmetry of fracture toughness," Phys. Rev. Lett. 126, 025503 (2021). 